\title{
TORCH: a large-area detector for high resolution time-of-flight
}

\author{
R. Forty ${ }^{1}$, N. Brook ${ }^{2}$, L. Castillo García ${ }^{3}$, D. Cussans ${ }^{4}$, K. Föhl ${ }^{1}$, C. Frei ${ }^{1}$, \\ R. Gao ${ }^{3}$, T. Gys ${ }^{1}$, N. Harnew ${ }^{3}$, D. Piedigrossi ${ }^{1}$, J. Rademacker ${ }^{4}$, \\ A. Ros García ${ }^{4}$, and M. van Dijk ${ }^{1}$ \\ 1 CERN, CH-1211 Geneva, Switzerland \\ Roger.Forty@cern.ch \\ 2 University of Bath, BA2 7AY, United Kingdom \\ 3 University of Oxford, OX13RH, United Kingdom \\ 4 University of Bristol, BS8 1TL, United Kingdom
}

\begin{abstract}
TORCH is a novel detector concept for high resolution timeof-flight measurement over large areas, which has been developed for application in a future upgrade of the LHCb experiment. The status of the $R \& D$ project is presented, including the development of suitable fast photon detectors, and test-beam studies of prototypes.
\end{abstract}

Keywords: particle identification, time-of-flight, photon detectors, $\mathrm{LHCb}$

\section{TORCH detector concept}

TORCH (Timing Of internally Reflected CHerenkov light) is an evolution of the DIRC technique, adding precision timing and angular information. It uses a highly polished plate of synthetic quartz as Cherenkov radiator (1-cm thick, $\left.\sim 8 \% X_{0}\right)$. When traversed by a charged particle, promptly produced Cherenkov photons are trapped in the plate and propagate to its edge by total internal reflection. The innovation is to measure, along with the detected photon hit positions, also their direction of propagation. This is achieved by coupling a focusing block to the edge of the radiator plate, as shown in Fig. 1, and using an extended plate (rather than bar) of quartz. Related developments have been pursued for the Belle II and PANDA experiments [1].

When a detected photon is correctly matched to the charged particle track that emitted it, the distance of propagation can then be determined. By measuring the Cherenkov angle at emission, the wavelength of photon can also be calculated, to correct for dispersion in the quartz. Fast photon detectors are used: micro-channel plate photomultipliers (MCP-PMT). The target for their intrinsic timing resolution is 50 ps per detected photon. The focusing scheme requires a linear array of detectors, with fine pixellization in one direction, coarse in the other. For 2-inch tubes $(60 \mathrm{~mm}$ pitch) the pixellization should be $128 \times 8$, i.e. $0.4 \times 6.6 \mathrm{~mm}^{2}$ pixels, to give an angular resolution of $\sim 1 \mathrm{mrad}$ in both projections. This gives a contribution to the resolution of $50 \mathrm{ps}$, leading to a total 

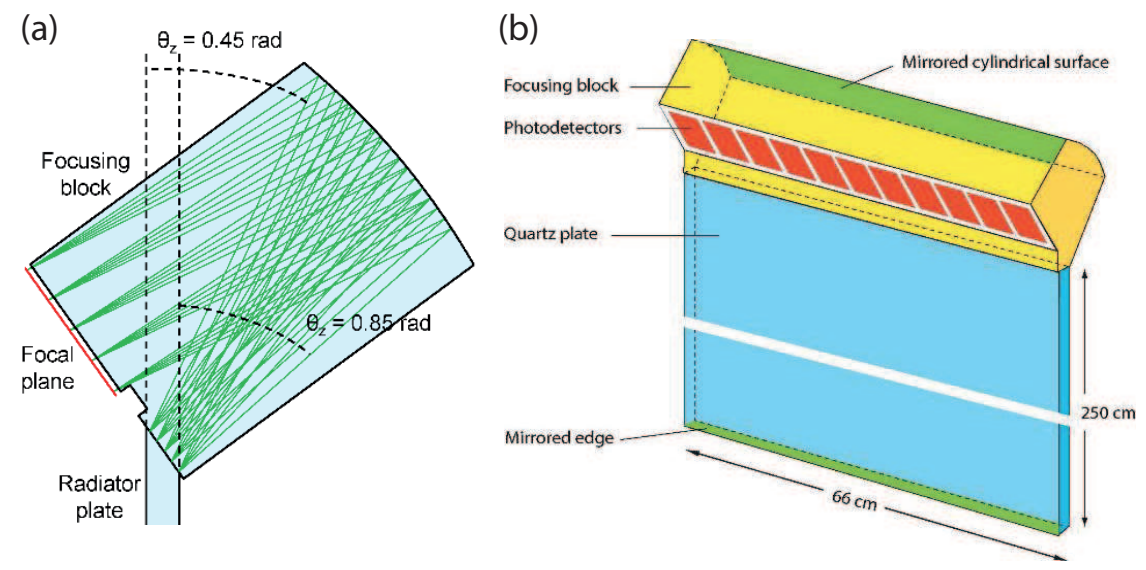

Fig. 1. (a) Cross-section through the focusing block attached to the edge of the radiator plate, illustrating the focusing of photons emitted from the plate. (b) Schematic view of a TORCH module.

resolution per detected photon of 50 (intrinsic) $\oplus 50$ (pixel size) ps $=70$ ps. For 30 detected photons per track (under the assumption that they are uncorrelated) this would provide $70 / \sqrt{30}=15$ ps resolution on the arrival time of the track at the TORCH detector.

\section{Application in $\mathrm{LHCb}$}

$\mathrm{LHCb}$ is the dedicated flavour physics experiment at the LHC, studying CP violation and rare decays of beauty and charm hadrons. It is a forward spectrometer, although operated in pp collider mode. An upgrade in preparation for 2019-20, to move to a fully software trigger, reading out the detector at the bunch-crossing rate of $40 \mathrm{MHz}$, with luminosity levelled at $2 \times 10^{33} \mathrm{~cm}^{-2} \mathrm{~s}^{-1}$. A further "Phase-II" upgrade is now under discussion, to push the luminosity further towards what is available from the LHC in the HL-LHC era (from 2024 onwards) [2].

Particle identification (in particular, distinguishing the charged hadrons $\mathrm{p}, \mathrm{K}$ and $\pi$ ) is crucial for much of hadronic physics of LHCb, and is currently provided by a RICH system. Low-momentum particle ID was previously provided by an aerogel radiator, but this was not suitable for the higher occupancy expected in the upgrade, and so has been removed. There is therefore currently no positive ID below the kaon threshold in the $\mathrm{C}_{4} \mathrm{~F}_{10}$ gas radiator of the $\mathrm{RICH}$, at $\sim 10 \mathrm{GeV} / c$. The difference in time-of-flight (TOF) between $\pi$ and $\mathrm{K}$ over $10 \mathrm{~m}$ is $\sim 40 \mathrm{ps}$ at $10 \mathrm{GeV} / c$, so 15 ps resolution would provide clear $(\sim 3 \sigma)$ separation.

A "start" time is needed for the TOF measurement: it could be provided by the accelerator clock, but would need to be corrected for the timing spread in the beam bunches. An alternative is to use signals from other tracks in the 
(a)

(b)

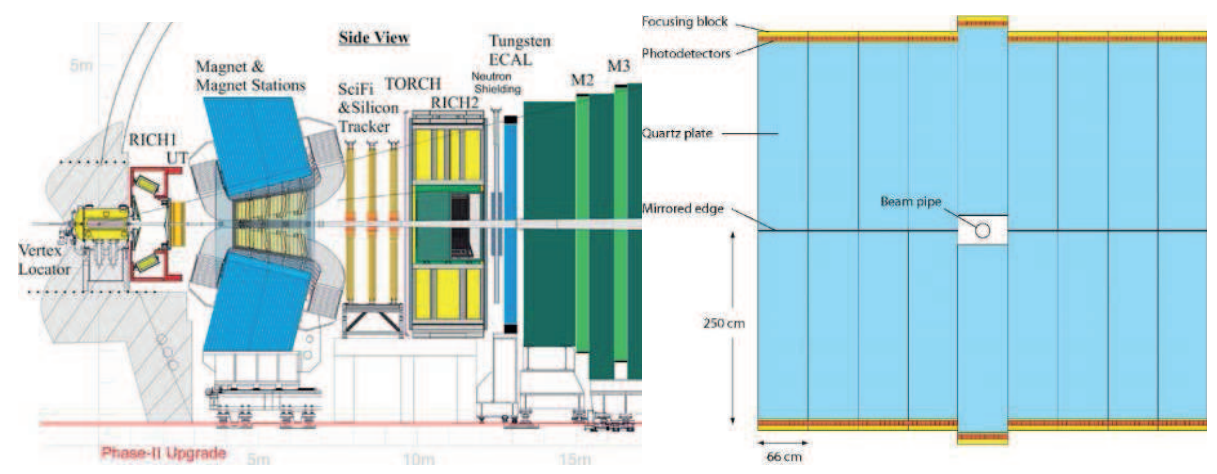

Fig. 2. (a) Layout of the LHCb spectrometer along the beam axis, as proposed for the Phase-II upgrade [2]; the TORCH detector is sited just after the main tracker. (b) Transverse layout of TORCH modules in $\mathrm{LHCb}$, to cover the full acceptance.

event from the primary vertex, in the TORCH detector itself. Typically most are pions, so the reconstruction logic can be reversed, and the start time is determined assuming they are all $\pi$, after removing outliers from other particles. In this way a few-ps resolution can be achieved on the start time.

At the foreseen location in $\mathrm{LHCb}(10 \mathrm{~m}$ from the interaction point) an area of $5 \times 6 \mathrm{~m}^{2}$ has to be covered, which is not feasible with a single plate, and anyway an aperture is required for the beam pipe. It is proposed to tile the surface using 18 identical modules (each $66 \times 250 \mathrm{~cm}^{2}$ ), as shown in Fig. 2. This will require 198 photon detector tubes, with $\sim 100 \mathrm{k}$ channels in total. Reflections from the transverse edges of modules will lead to ambiguities in the reconstruction, but at a level that can be resolved by the pattern recognition. At the luminosity expected in the upgrade of LHCb there will be a high track multiplicity, of over 100 charged tracks per event, but the performance of TORCH has been studied with simulation in these conditions and is excellent. Fast timing will also be very useful for pile-up suppression at high luminosity, as is being explored by the other experiments at the LHC [3].

\section{Status of the $R \& D$ project}

An EU-funded R\&D project for TORCH has been running for five years, to develop suitable photon detectors and provide proof-of-principle with a prototype module. The project is a collaboration with industrial partner Photek (UK).

At the start of the project, commercial tubes were not available that satisfied all the requirements of TORCH:

1. fast timing ( $<50$ ps per detected photon);

2. high active area ( $>80 \%$ for the linear array);

3. fine pixellization $\left(128 \times 8\right.$ rectangular pixels in a $60 \times 60 \mathrm{~mm}^{2}$ tube); 
(a)

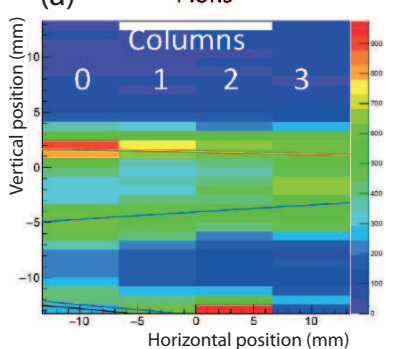

(c) (b) Protons

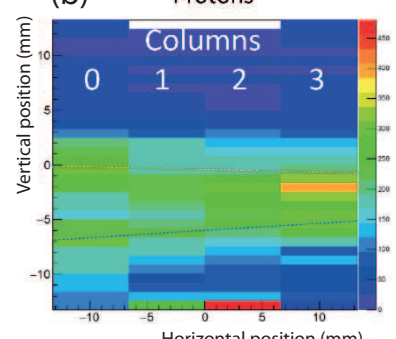

(d)
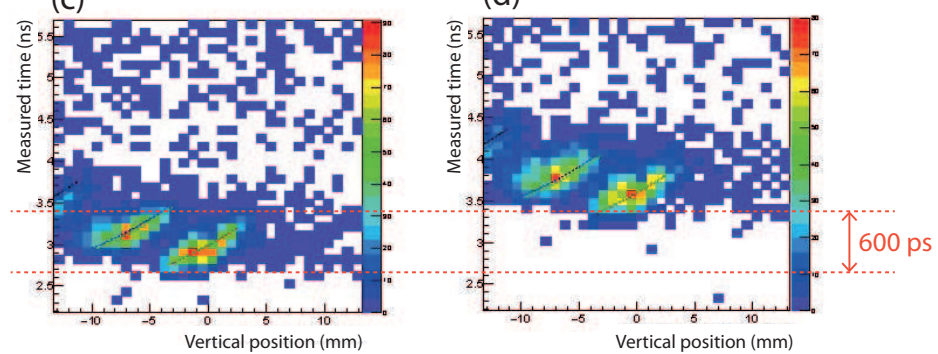

Fig. 3. Data from the test beam: hit pattern in the photon detector for selected pions (a) and protons (b); reconstructed time distribution versus vertical position in one of the columns of pixels in the photon detector for pions (c) and protons (d).

4. long lifetime (up to $5 \mathrm{C} / \mathrm{cm}^{2}$ charge density at the anode).

A three-phase $R \& D$ program has been followed, to develop these characteristics separately, and then bring them together in a final prototype tube [4].

The intrinsic timing performance of the first prototype tubes was measured with a fast laser and single-channel commercial readout electronics. The tubes have a dual-MCP in chevron configuration, with $10 \mu \mathrm{m}$ pores. A resolution of 23 ps has been achieved, with a small tail from laser and back-scattering effects [5]. The lifetime issue was addressed by ALD (atomic layer deposition) treatment of the MCP, as pioneered at Argonne/LAPPD [6], and tubes have been successfully tested up to an integrated charge density at the anode of $4 \mathrm{C} / \mathrm{cm}^{2}[7]$.

Custom readout electronics have been developed, based on a chip set originally developed for the ALICE TOF detector [8]. The 32-channel NINO chip provides fast amplification and time-over-threshold as an estimate of input charge, and the HPTDC chip performs digitization (with 100 ps bins) [9]. An effective resolution equivalent to $0.4 \mathrm{~mm}$ can be achieved with two-times larger pixels by making use of charge-sharing between neighbouring pixels. The point-spread function was adjusted in the second phase of prototype tubes to share charge over 2-3 pixels. Calibration of the relationship between pulse width and charge was performed, and the spatial resolution measured with laser illumination (using the charge-weighted cluster centroid), to be better than $100 \mu \mathrm{m}$ [10].

The final prototype tube integrates the features that have been developed in the earlier phases, in a square format with $53 \times 53 \mathrm{~mm}^{2}$ active area. It features 

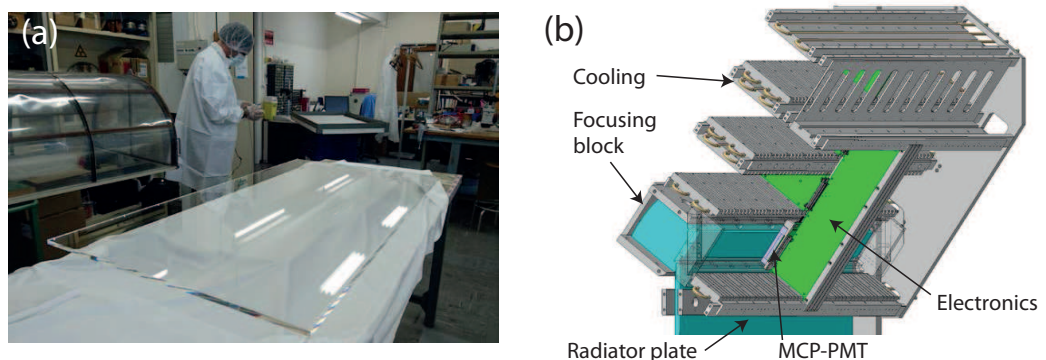

Fig. 4. (a) Radiator plate for the large prototype, after delivery to CERN. (b) Detail of the design for the large prototype, indicating the various components mounted at the upper edge of the radiator plate.

a quartz window and AC-coupled anode, so the window can be kept at ground potential. Readout connectors are mounted on a $\mathrm{PCB}, 64 \times 8$ pixels per tube, which is attached to tube using ACF (anisotropic conductive film). The delivery of final tubes from Photek is scheduled during Summer 2017.

\section{Test-beam studies}

A small prototype module has been constructed for beam tests at the CERN PS-T9 area. Optical components were delivered by Schott, with a second-phase prototype MCP-PMT from Photek. The radiator plate is $35 \times 12 \times 1 \mathrm{~cm}^{3}$, and was coupled to the focusing block using silicone (Pactan 8030). The time-of-flight could be independently determined using dedicated timing stations $10 \mathrm{~m}$ apart, which allowed the $\mathrm{p} / \pi$ components of the beam to be separated. The detected hits seen in the MCP-PMT match the expected pattern (taking into account reflections from edges), as shown in Fig. 3. The small difference in Cherenkov angle for $\pi$ and $\mathrm{p}$ at $5 \mathrm{GeV} / c$ is visible comparing (a) and (b). The time measured for each cluster is plotted versus vertical position along one column of pixels in (c) and (d), and the reflections are clearly separated. The $\mathrm{p}-\pi$ time-of-flight difference of about $600 \mathrm{ps}$ is cleanly resolved.

Projecting along the timing axis relative to the prediction for the earliest pion signal, for each column of pixels (using the nearest timing station as reference) gives a core distribution with $\sigma \approx 110 \mathrm{ps}$. This is before subtraction of the contribution from the timing reference itself, so we are approaching the target resolution of $70 \mathrm{ps} /$ photon. Small tails seen in the timing distribution are due to imperfections in the calibration and back-scattering effects.

A large prototype of a TORCH module on the scale that would be required for $\mathrm{LHCb}$ is now under construction, with full width and half height: $125 \times 66 \times 1 \mathrm{~cm}^{3}$. It will be equipped with $10 \mathrm{MCP}-\mathrm{PMTs}$, for a total of over 5000 channels. Optical components have been delivered by Nikon, see Fig. 4. Detailed measurements provided by the supplier match the specifications. This final deliverable of the $\mathrm{R} \& \mathrm{D}$ project will be ready for testing in beam over the next year. 


\section{Conclusions}

The TORCH detector concept adds precise angular and timing information to a DIRC, to provide high-precision time-of-flight over large areas. It is included in the plans for a future upgrade of the LHCb experiment. Suitable fast photon detectors have been developed with industry, with final prototypes expected to be delivered imminently. Test-beam studies have achieved close to the nominal performance, and a full-scale prototype module is under construction for testing over the next year: its success should provide a solid foundation for proposing the full detector in LHCb. It is an exciting time for the project!

\section{Acknowledgments}

Support from the European Research Council in funding the TORCH R\&D project is gratefully acknowledged (ERC-2011-AdG 291175-TORCH). The authors also thank the industrial partner Photek Ltd.

\section{References}

1. G. Varner (Belle II), M. Schmidt (PANDA), presentations at this conference.

2. Expression of Interest for a Phase-II upgrade of LHCb, CERN-LHCC-2017-003.

3. B. Lenzi (ATLAS), A. Bornheim (CMS), presentations at this conference.

4. J. Milnes (Photek), presentation at this conference.

5. T. Gys et al., NIM A766 (2014) 171.

6. C. Craven (Incom), presentation at this conference.

7. T. Gys, presentation at RICH 2016, Bled.

8. F. Anghinolfi et al., NIM A533 (2004) 183.

9. R. Gao et al., JINST 10 C02028 (2015).

10. L. Castillo García, JINST 12 C02005 (2017). 